\title{
Effects of Different Taping Pressures with Wrist Taping on Isokinetic Strength Exertion of Wrist Dorsal and Palmar Flexion
}

\author{
${\text { Kenji Takahashi }{ }^{1, *} \text {, Shin-ichi Demura }}^{2}$ \\ ${ }^{1}$ Department of Judo Physical Therapy, Teikyo Heisei University, Uruidominami 4-1 Ichihara, chiba, Japan \\ ${ }^{2}$ Graduate School of Natural Science \& Technology, Kanazawa University, Kakuma, Kanazawa, Ishikawa, Japan \\ *Corresponding author: kenji.takahashi@thu.ac.jp
}

Received December 17, 2014; Revised December 21, 2014; Accepted December 24, 2014

\begin{abstract}
We aimed to examine the effects of wrist taping at different pressure levels on isokinetic strength exertion of dorsal and palmar flexion. Nineteen healthy male university students were enrolled. The wrist-taping method involved winding a rigid tape around wrist joint thrice. A qualified athletic trainer adjusted taping pressures using a pressure measuring system, whose sensor was on the palmaris longus muscle tendon of the dominant wrist. Isokinetic dorsal and palmar flexion strength was measured by an isokinetic dynamometer system. Taping pressure [5, 30, 60, and $90 \mathrm{hPa}$ and control (no tape)] and angular velocity [slow (60\%)/sec), moderate (180\% $/ \mathrm{sec})$, and fast $(300 \% \mathrm{sec})]$ were considered independent variables. Peak torque $(\mathrm{Nm})$ of isokinetic strength exertion was considered the dependent variable. Two-way repeated measures analysis of variance (taping pressure $\times$ angular velocity) was used to calculate the mean differences for peak torque conditions. A significant difference was found only in the main effect of angular velocity. Multiple comparison tests showed that the isokinetic strength exertion was largest in fast flexion in all taping pressure conditions for dorsal flexion, whereas it was largestin slow flexion in the control and 5-hPa conditions for palmar flexion. For palmar flexion, it was larger in slow flexion for $<5$-hPa taping pressure, but not for $>30-\mathrm{hPa}$. The effects of taping pressure and flexion speed on isokinetic strength exertion may differ between dorsal and palmar flexion of the same wrist.
\end{abstract}

Keywords: competitive sports, angular velocity, palmaris longus muscle tendon, wrist injury, range of motion

Cite This Article: Kenji Takahashi, and Shin-ichi Demura, "Effects of Different Taping Pressures with Wrist Taping on Isokinetic Strength Exertion of Wrist Dorsal and Palmar Flexion.” American Journal of Sports Science and Medicine, vol. 2, no. 6 (2014): 222-226. doi: 10.12691/ajssm-2-6-4.

\section{Introduction}

Athletes such as sumo fighters, wrestlers, judo competitors, weightlifters, and gymnasts fix wrist joints using taping or special splint to prevent wrist disorders or injuries by limiting excessive wrist motion [1,2]. However, fixed taping has been reported to decrease strength exertion and motor performance parameters. According to Rettig et al. [3] a taping method that fixes fingers and the wrist joint decreases grip strength exertion. Kauranen et al. [4] reported that wrist taping induces decreased motor performance parameters, such as simple reaction time, choice reaction time, and tapping speed, as well as decreased isokinetic strength exertion during wrist palmar and ulnar flexion. In competitive sports, limitation of strength exertion related to competitive behaviors negatively affects performance. Henceit is unclear whether use of wrist taping in situations such as competitive matches and training is useful.

Constantinou and Brown [1] pointed out the necessity for control taping pressure after reviewing studies analyzing the effects of taping. Even when using the same taping method, effects on the strength exertion may be different according to varying taping pressures. Takahashi et al. [5] reported that wrist taping pressure of $>30 \mathrm{hPa}$ with elastic tape slightly limits grip strength exertion. However, the effect of wrist taping with varying taping pressure on isokinetic strength exertion has not yet been examined. On the basis of Takahashi et al.'s result, we hypothesized that wrist taping with pressure decreases isokinetic strength exertion.

Kauranen et al. [4] reported that wrist taping decreases isokinetic strength exertion of palmar and ulnar flexion at a moderate angular velocity $\left(180^{\circ} / \mathrm{sec}\right)$; however, they found that isokinetic strength exertion was unchanged in all wrist joint movements at a slow angular velocity $(60 \% \mathrm{sec})$. In short, the effect was shown to vary according to angular velocity. The range of palmar flexion is greater than that of dorsal flexion, and the range of ulnar flexion is greater than that of radial flexion [6]. Thus, isokinetic strength exertion may be limited in cases requiring a large range of motion. From the above, we hypothesized the following: isokinetic strength exertion decreases more in palmar flexion than in dorsal flexion, and this effect increases in the order of slow, moderate, and fast angular velocities. 
This study aimed to examine the effects of wrist taping with different pressure on the isokinetic strength of dorsal and palmar flexion with slow, moderate, and fast angular velocities.

\section{Methods}

\subsection{Subjects}

Subjects were 19 male university students (mean age, $20.6 \pm 0.9$ years) with an athletic experience of $>5$ years and without history of wrist injuries such as sprains, fractures, or dislocations. These athletes participated in the following sport events: baseball $(n=8)$, soccer $(n=2)$, badminton $(n=2)$, basketball $(n=2)$, long-distance running in track and field $(\mathrm{n}=1)$, throwing in track and field $(\mathrm{n}=1)$, volleyball $(n=1)$, handball $(n=1)$, and kendo $(n=1)$. Table 1 shows the basic characteristics [age, height, body weight, body mass index (BMI), wrist circumference, and competitive sports experience] of the subjects.

The aim and procedures of this study were explained in detail to all subjects before the experiments, and written informed consent was obtained. This experimental protocol was approved by the Ethics Committee on Human Experimentation of the Faculty of Human Science, Kanazawa University (2012-18).

Table 1. The basic statistics for age, height, body weight, BMI and circumference of the dominant wrist joint of subjects $(n=19)$

\begin{tabular}{ccccc}
\hline & Mean & SD & MAX & MIN \\
\hline Age (years) & 20.6 & 0.9 & 22 & 19 \\
Height (cm) & 170.5 & 6.2 & 184.3 & 158.4 \\
Weight (kg) & 64.4 & 9.0 & 85.2 & 50.0 \\
BMI & 22.2 & 3.3 & 32.3 & 18.7 \\
Wrist circumference of dominant hand & 15.9 & 0.9 & 17.5 & 14.2 \\
(cm) & 9.3 & 3.4 & 18.0 & 5.0 \\
\hline Sports experience (years) & & & & \\
\hline
\end{tabular}

\subsection{Taping Performer}

Wilson et al. [7] and Pfeiffer et al. [8] controlled the taping effect depended on performers by employing a qualified trainer. Hence, in our study, a qualified athletic trainer (certified by the Japan Sport Association)with clinical experience of $>10$ years performed the taping.

\subsection{Devices}

\subsubsection{Tape \& Taping Method}

In this study, we used 50-mm rigid (nonelastic) tape manufactured by Johnson \& Johnson (New Brunswick, NJ, USA). We adopted the wrist taping method that wound one piece of rigid tape thrice around the wrist joint, including the radial and ulnar styloid processes[5].

\subsubsection{Pressure-measuring Device}

Taping pressure was measured using the pressure measuring system for stockings and bandages (AMI3037SB; AMI-Techno, Tokyo, Japan). This system can measure pressure on the human body through clothing such as socks [9] and can detect the addition of constant pressure such as that from the use of an elastic bandage [10]. Therefore, this device is useful in measuring taping pressure. A measurable range was $1-200 \mathrm{hPa}$, and the measurement error was $\pm 3 \mathrm{hPa}$.

\subsubsection{Isokinetic Strength-measuring Device}

Isokinetic strength (expressed in $\mathrm{Nm}$ ) of dorsal and palmar flexion was measured using an isokinetic Biodex ${ }^{\circledR}$ dynamometer system 4 (Biodex Corp., Shirley, NY, USA). The subjects were positioned in the following sitting posture, based on the manufacturer's manual: shoulder joint in $20^{\circ}-30^{\circ}$ forward flexion and $20^{\circ}-30^{\circ}$ abduction, elbow joint in $70^{\circ}-80^{\circ}$ flexion, and forearm in full pronation.

Three angular velocities in the measurement were set in the following three conditions: slow $(60 \% \mathrm{sec})$, moderate $\left(180^{\circ} / \mathrm{sec}\right)$ (according to the experiments of Kauranen et al. [4]) and fast $\left(300^{\circ} / \mathrm{sec}\right)$. The movement range of the wrist joint was restricted between $30^{\circ}$ of dorsal flexion and $45^{\circ}$ of palmar flexion to ensure the safety of the athletes. In short, subjects repeated movement range (a total of $75^{\circ}$ ) of dorsal and palmar flexion for a specified number of repetitions during the measurements.

\subsection{Independent and Dependent Variables}

The independent variables were taping pressure and angular velocity. The former consisted of five conditions [5 hPa, $30 \mathrm{hPa}, 60 \mathrm{hPa}$, and $90 \mathrm{hPa}$, and control (no tape)], according to Takahashi et al. [5] The latter consisted of three conditions [slow $(60 \% \mathrm{sec})$, moderate $(180 \% / \mathrm{sec})$, and fast $(300 \%$ sec) $]$. Peak torque $(\mathrm{Nm})$ during isokinetic strength of dorsal and palmar flexion was considered the dependent variable.

\subsection{Procedure}

Isokinetic strength measurement and wrist taping were performed on the wrist joint of the dominant hand, with the hand dominance being decided according to Demura's handedness inquiry [11]; all subjects had right hand dominance. Before the experiment, they performed wrist warm-up exercises to prevent injury. A pressure sensor was placed over the palmaris longus muscle tendon $1.5 \mathrm{~cm}$ proximal to the palmar crease of the wrist, and was fixed with a cover-tape, after which the wrist taping was performed(Figure 1a, 1b, and 1c).Taping pressure was adjusted while monitoring values using the pressuremeasuring device [10]. Because the initial taping pressure could not be exactly measured, the error range for $5-\mathrm{hPa}$ condition was within $\pm 1 \mathrm{hPa}$ and that for the other pressure conditions was within $\pm 2 \mathrm{hPa}$.

Isokinetic strength was measured in the order of fast, moderate, and slow angular velocities because we confirmed by a preliminary experiment that the load to wrist joint becomes stronger as angular velocity becomes slower. Movements were repeated six times in fast angular velocity condition as well as three times in moderate and slow angular velocity conditions, to factor in the time that subjects needed to get to measurement movements. The rest period between each angular velocity condition was 5 min. Readjustments of the taping pressure were made during the 5-min rest period.

Taping pressure conditions and control condition were randomized for every subject, and each subject underwent measurements in only one condition on a day. A practice trial was performed on the day before the experiment. The experiment was performed during 9:00-13:00 in a laboratory, with the ambient temperature being maintained at $26^{\circ} \mathrm{C}$. 


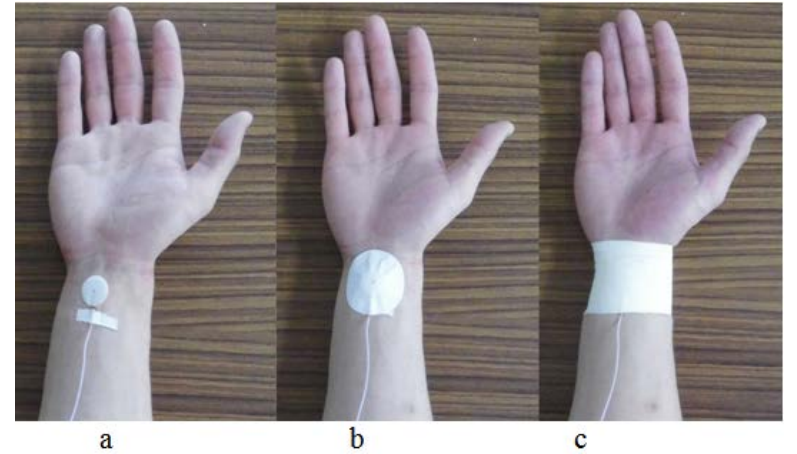

Figure 1. Pressure sensor is set on the palmaris longus muscle tendon a: pressure sensor only

b: pressure sensor + cover tape

c: pressure sensor + cover tape + wrist taping

\subsection{Statistical Analysis}

Two-way repeated measures analysis of variance (taping pressure $\times$ angular velocity) was used to reveal the mean differences among each condition for the peak torque during dorsal and palmar flexion. When significant effects were found, a multiple comparison test was performed using the Tukey's honestly significant difference (HSD) method. The level of significance was set at 0.05. In addition, an effect size (ES) was calculated to examine the size of mean differences. ES was interpreted as follows: $<0.2$ as small, $>0.5$ as moderate, and $>0.8$ as large.

\section{Results}

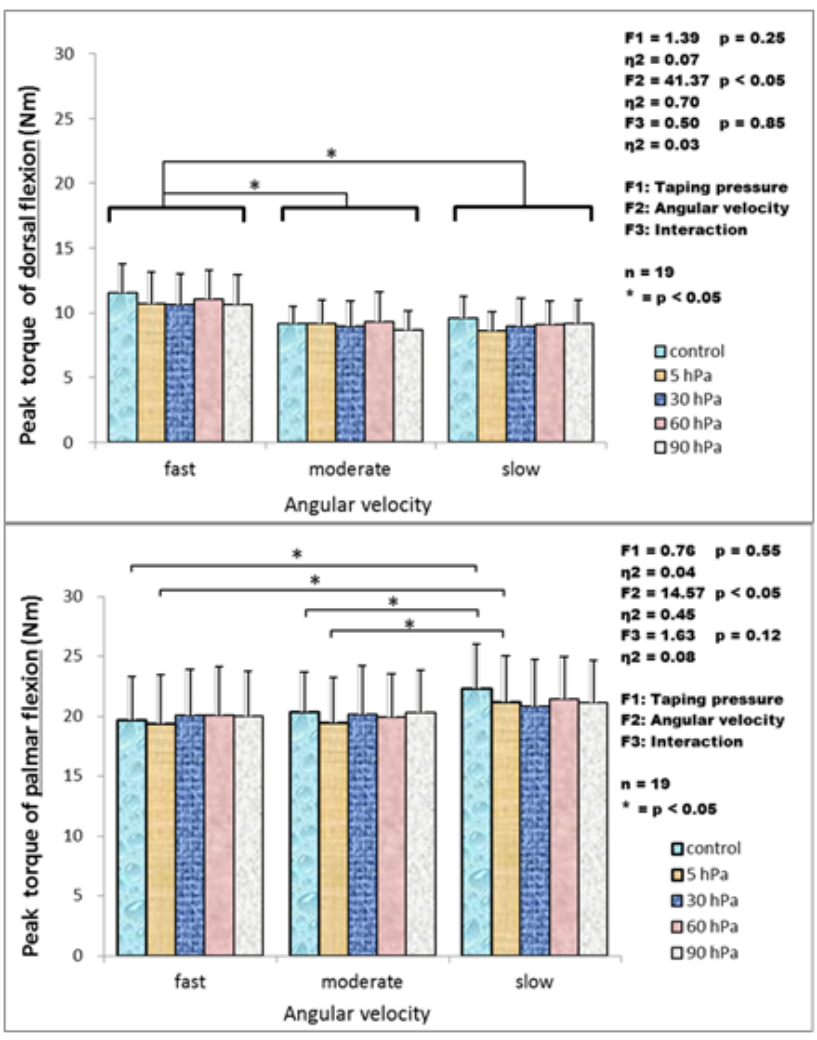

Figure 2. Means and standard deviations of peak torque for wrist dorsal flexion (above) and palmer flexion (below), and the results of two-way repeated measures analysis of variance.
Figure 2 show the means and standard deviations of the peak torque under all experimental conditions, and the results of the two-way repeated measures analysis of variance (taping pressure $\times$ angular velocity) for dorsal and palmar flexion, respectively. A significant difference was found only in the main effect of angular velocity factor for both movements. Multiple comparison tests showed that for dorsal flexion, isokinetic strength exertion was significantly larger with fast angular velocity than with moderate and slow velocities in all taping pressure conditions (ES: 0.71-1.28). With regard to palmar flexion, isokinetic strength exertion was significantly larger with slow angular velocity than with fast or moderate angular velocity in the control and $5-\mathrm{hPa}$ taping pressure conditions (ES: 0.46-0.72); however, a significant difference was not found among velocity conditions for taping pressures of $>30 \mathrm{hPa}$.

\section{Discussion}

This study aimed to examine the effects of wrist taping with different pressures on the isokinetic strength of dorsal and palmar flexion. Kauranen et al. [4] reported that wrist taping limited isokinetic strength exertion during palmarflexion. However, they did not specify the taping pressure used.

\subsection{Effects of Taping Pressures}

We hypothesized that wrist taping with the pressure limited isokinetic strength exertion, on the basis of Takahashi's report [5] that wrist taping at pressure $>30$ $\mathrm{hPa}$ with elastic tape slightly limited grip strength exertion. However, a limitation effect of strength exertion by difference taping pressure of wrist taping could not be confirmed for both wrist palmar and dorsal flexion in this study. In short, the above hypothesis was rejected. Miyamoto et al. [12] reported that wearing the elastic stocking at a pressure of $30 \mathrm{mmHg}$ over ankle joints did not affect strength exertion on calf raise exercise. Considering this, our finding that wrist taping does not affect dynamic strength exertion may be accurate.

Some athletes expect to exert larger strength by the wrist taping [3]. This idea is based on the fact by enhancing the stability of joints by wrist taping, the athletes can focus on the target action without dispersing the force vector [13]. However, isokinetic strength exertion also did not increase in the present study; this was in agreement with the findings of Rettig et al. [3]. Hence, strength exertion cannot be expected to increase with wrist taping.

\subsection{Differences between Dorsal and Palmar Flexion}

Kauranen et al. [4] reported that isokinetic strength exertion during palmar flexion was limited by wrist taping. Because the range of palmar flexion of the wrist joint is larger than that of dorsal flexion, this difference is considered to influence the effect of wrist taping on strength exertion. Thus, we hypothesized that the isokinetic strength exertion is more limited in palmar flexion than in dorsal flexion. However, this hypothesis 
was also rejected. In short, we were unable to confirm the limitation of strength exertion in both movements.

Mochizuki [14] reported that midcarpal and radiocarpal joints move at the same rate until $30^{\circ}$ dorsal flexion and $40^{\circ}$ palmar flexion, beyond which it is the midcarpal joint that mainly accounts for the movements. In the present study, we measured strength exerted within a range of $30^{\circ}$ dorsal flexion and $45^{\circ}$ palmar flexion. Hence, it is possible that the effect of taping was not observed in both motions because of a small measurement range. Although the effect of taping may be observed by enlarging measurement ranges, this would predispose to injuries because of increased load on the wrist joint. Hence, it is necessary to conduct experiments carefully and safely.

\subsection{Effect of Angular Velocity}

We hypothesized that the limitation effect of isokinetic strength exertion by wrist taping becomes stronger as the angular velocity becomes faster, according to the results of Kauranen's study [4]. However, this hypothesis was also rejected. In short, the strength exertion was not limited even in faster angular velocities.

For dorsal flexion, the peak torque was the largest in fast angular velocity in all taping pressure conditions. For palmar flexion, a significant difference among velocities was found in the control and 5-hPa taping pressure conditions, but not with taping pressures of $>30 \mathrm{hPa}$. Thus, for palmar flexion, force exertion may not be limited by the angular velocity of the wrist in taping pressures of $>30$ $\mathrm{hPa}$.

Kauranen et al. [4] and Ellenbecker et al. [15] reported that for junior elite tennis players, the peak torque of both wrist movements was larger with slow angular velocity than with moderate angular velocity. In addition, peak torque for the knee joints also decreases with increasing angular velocity $[16,17]$. Thus, the peak torque may generally show a high value in slow angular velocities. The results of the present study showed a similar trend with palmar flexion, but not with dorsal flexion. Pain during measurements is considered as one of the causes: in the preliminary experiments, most subjects complained of wrist pain at slow angular velocities and some in moderate angular velocity. Thus, it is considered that wrist pain limited the isokinetic strength exertion. Because the wrist joint is smaller than shoulder and knee joints, the pain is easily induced when imposing a large load; thus, wrist pain may be larger if large loads are imposed in with slow angular velocities.

On the other hand, no subjects complained of wrist pain with palmar flexion. Because palmar flexion is used more frequently than dorsal flexion in competitive sports and daily life, wrist pain may have been infrequent during measurements irrespective of motion speeds. We believe that most subjects were not familiar with dorsal flexion activities because they did not frequently use it in sporting activities. In future, it will be necessary to examine the isokinetic strength exertion during dorsal flexion in competitive athletes using frequently dorsal flexion.

\section{Conclusion}

For dorsal flexion, irrespective of a difference in taping pressures, isokinetic strength exertion is larger in fast movements than in moderate and slow movements. For palmar flexion, isokinetic strength exertion is largest in slow movements with taping pressures of $<5 \mathrm{hPa}$, but it was not affected by flexion speed with taping pressures of $>30 \mathrm{hPa}$. Thus, even for the same wrist, the effects of taping pressure and flexion speed on isokinetic strength exertion differ considerably between dorsal flexion and palmar flexion movements.

\subsection{Practical Implications}

Wrist taping with pressure does not limit isokinetic strength exertion irrespective of motion speeds.

For palmar flexion, wrist taping with taping pressure of $>30 \mathrm{hPa}$ may suppress the decrease of peak torque because of the faster motion speed.

Using wrist taping with the pressure in situations such as competitive matches and training is useful because it does not affected dynamic strength exertion negatively and may prevent injury.

\section{Acknowledgment}

The authors would like to thank Enago (www.enago.jp) for the English language review.

\section{References}

[1] Constantinou M, Brown M. Therapeutic taping for musculoskeletal conditions. Elsevier, Churchill Livingstone, 2010.

[2] RoseM. Pocketbook of Taping Techniques, 1st ed., Elsevier, Churchill Livingstone 2009.

[3] Rettig A, Stube K, Shelbourne K. Effects of finger and wrist taping on grip strength. Am J Sports Med 1997; 25 (1): 96-98.

[4] Kauranen K, Siira P, Vanharanta H. The effect of strapping on the motor performance of the ankle and wrist joints. Scand J Med Sci Sports 1997; 7: 238-243.

[5] Takahashi K, Demura S, Noguchi Tet al.Effects of elastic wrist taping on maximum grip strength. Am J Sports Sci and Med 2013; 1 (3): 33-36.

[6] Norkin CC, White DJ. Measurement of Joint Motion: Guide to Goniometry, 4th ed., F.A. Davis Company, 2009.

[7] Wilson T, Carter N, Thomas G. A multicenter, single-masked of medial, neutral, and lateral patellar taping in individuals with patellofemoral pain syndrome. J Orthop Sports Phys Ther 2003; 33 (8): 437-448.

[8] Pfeiffer RP, DeBeliso $M$, Shea KG et al. Kinematic MRI assessment of McConnell taping before and after exercise. Am J Sports Med2004; 32 (3): 621-628.

[9] Ooizumi Y, Matsuzawa E, Iida Kenichi. Establishment of evaluation methods of clothing pressure of high supported clothesThe relation between clothing pressure of stretch clothes measured on a dummy and the human body [in Japanese]. Bulletin of TIRI 2007; 2: 120-121.

[10] Hirai M. Clinical application of elastic stockings [in Japanese]. Japanese Journal of Phlebology 2007; 18 (5): 239-245.

[11] Demura S, Sato S, and Nagasawa Y. Re-examination of useful items for determining hand dominance [in Japanese]. Arch Sci Med (Torino) 2009; 168: 169-177.

[12] Miyamoto N, Hirata K, Mitsukawa N et al. Effect of pressure intensity of graduated elastic compression stocking on muscle fatigue following calf-raise exercise. $J$ Electromyogr Kinesiol 2011; 21 (2): 249-254.

[13] Weijie F, Yu L, Songning Z et al. Effects of local elastic compression on muscle strength, electromyographic, and mechanomyographic responses in the lower extremity. $J$ Electromyogr Kinesiol 2012; 22 (1): 44-50.

[14] Mochizuki Y. Experimental study on the kinematics of the wrist joint [in Japanese]. Hiroshima Daigaku Igaku Zasshi 1992; 39 (1): 105-126. 
[15] Ellenbecker TS, Roetert EP, Riewald S. Isokinetic profile of wrist and forearm strength in elite female junior tennis players. $\mathrm{Br} J$ Sports Med 2006; 40 (5): 411-414.

[16] Ergün M, İşlegen Ç, Taşkıran E. A cross-sectional analysis of sagittal knee laxity and isokinetic muscle strength in soccer players. Int J Sports Med 2004; 25 (8): 594-598.
[17] Yamaji S, Demura S, Nagasawa Yet al. The effects of kinesio taping on isokinetic muscle exertions of lower limb [in Japanese]. Jpn J PhysFitness Sports Med 1999; 48 (2): 281-289. 